\title{
Gallic acid suppresses colon cancer proliferation by inhibiting SRC and EGFR phosphorylation
}

\author{
XIAOMING LIN ${ }^{1 *}$, GUANGFEI WANG ${ }^{2 *}$, PING LIU ${ }^{2}$, LEI HAN ${ }^{2}$, \\ TONG WANG $^{2}$, KAILI CHEN ${ }^{2}$ and YONGLIN GAO ${ }^{2}$ \\ ${ }^{1}$ Luye Pharma Group Ltd., Yantai, Shandong 264000; ${ }^{2}$ School of Life Sciences, \\ Yantai University, Yantai, Shandong 264005, P.R. China
}

Received November 17, 2020; Accepted February 10, 2021

DOI: $10.3892 / \mathrm{etm} .2021 .10070$

\begin{abstract}
The aim of the present study was to investigate the effects of gallic acid (GA) on the proliferation and apoptosis of colon cancer cells and to further clarify the mechanism of GA function associated with SRC and EGFR phosphorylation. HCT116 and HT29 cells were treated with different concentrations of GA for $24 \mathrm{~h}$. Cell proliferation and apoptosis were analyzed using plate clone formation and flow cytometry assays, respectively. In addition, the expression of apoptosis-related proteins was examined by western blotting. Furthermore, the level of STAT3, AKT, SRC and EGFR phosphorylation was analyzed by western blotting and immunofluorescence. Subsequently, the SRC inhibitor PP2 and the EGFR inhibitor gefitinib were used to analyze the GA-associated mechanisms. In addition, a xenograft tumor model was established to confirm the effects of GA in vivo. The results indicated that GA inhibited cell proliferation and promoted cell apoptosis by upregulating the ratio of cleaved caspase-3/pro-caspase-3 and cleaved caspase-9/pro-caspase-9. Concurrently, GA decreased the level of phosphorylated (p)-SRC, p-EGFR, p-AKT and p-STAT3. Following treatment with PP2 and gefitinib in both cancer cell lines and animal model, GA was demonstrated to inhibit EGFR and SRC phosphorylation to downregulate STAT3 and AKT phosphorylation. In vivo, GA prevented tumor growth, promoted tumor apoptosis and decreased the level of p-SRC, p-EGFR, p-STAT3 and p-AKT. In conclusion, GA was indicated to suppress colon cancer proliferation by inhibiting SRC and EGFR phosphorylation.
\end{abstract}

Correspondence to: Professor Yonglin Gao, School of Life Sciences, Yantai University, 32 Qingquan Road, Laishan, Yantai, Shandong 264005, P.R. China

E-mail: gylbill@126.com

*Contributed equally

Key words: gallic acid, proto-oncogene tyrosine-protein kinase Src, epidermal growth factor receptor, signal transducer and activator of transcription 3, protein kinase B/AKT, colon cancer

\section{Introduction}

Colon cancer is a malignant tumor and is the third most common cancer worldwide which is expected to increase by $60 \%$ to $>2.2$ million new cases and 1.1 million deaths by 2030 (1). Colon cancer is a serious threat to public health due to its difficulty to operate on and its ability to easily metastasize (1,2). A number of factors may cause colon cancer, such as environment, lifestyle (including lack of exercise, which can lead to obesity) and eating habits (excessive intake of saturated fats and low vegetable and fruit intake) $(3,4)$. The current treatment of colon cancer mainly involves surgery, supplemented by chemotherapy and improved diet.Early diagnosis by endoscopy is a primary goal in diagnosing and treating colon cancer (5). Therefore, chemotherapy serves an important role in the clinical treatment of colon cancer (5).

Current commonly used chemotherapeutic drugs for colon cancer include 5-fluorouracil (5-FU) (6), oxaliplatin (6), capecitabine (7) and irinotecan (8). These drugs are cytotoxic, often due to insufficient selectivity of the drug to the site of colon cancer, resulting in a series of adverse reactions, such as neutropenia, anemia, diarrhea, gastrointestinal toxicity, mucositis, nausea and vomiting, as well as blood system diseases and liver toxicity (6-8). In addition, drug resistance may also develop, which seriously affects the quality of life and lifespan of patients (6-8). Compared with traditional chemotherapeutics, several natural biological compounds (9), including artemisinin, celastrol and resveratrol, present unique advantages, such as less toxicity, fewer side effects and pain relief for patients with tumors. Therefore, researchers have focused on discovering novel antitumor drugs from natural sources.

Plant extracts have demonstrated unique advantages in preventing colon cancer. For example, a previous study (10) revealed that piperine can affect HT29 cells, which is a colon cancer cell line, and inhibit tumor cell proliferation. Moreover, piperine has been indicated to increase the production of reactive oxygen species, activate endoplasmic reticulum emergency-associated proteins, inhibit the phosphorylation of AKT and JNK and activate p38 MAPK, which can eventually induce cells to undergo caspase-mediated mitochondrial apoptosis (10). Other studies have also demonstrated that anthocyanins are non-toxic to normal intestinal cell lines in vitro $(11,12)$, and can inhibit human colon cancer cell growth and induce apoptosis in a dose- and time-dependent manner (13). 
Gallic acid (GA) is a type of natural polyphenol compound that exists in nature, and it is widely found in several Chinese medicines, such as green tea, Ganoderma lucidum and other plants (14). GA has been indicated to exert important anti-inflammatory (15) and antiviral (16) effects. Of note, GA can inhibit the proliferation of tumor cells. For instance, several studies have indicated that GA exhibited an inhibitory effect on lung cancer and esophageal cancer cells $(17,18)$. However, to date, there are few systematic and comprehensive studies on GA in colon cancer, and the underlying mechanism needs to be further elucidated.

Therefore, the present study was designed to investigate whether GA could be used to inhibit the proliferation of colon cancer cells and determine the potential mechanisms via in vivo and in vitro experiments.

\section{Materials and methods}

Cells and grouping. The human colon cancer cell lines HCT116 (cat. no. BNCC337692) and HT29 (cat. no. BNCC100164) were purchased from BeNa Culture Collection, Beijing Beina Chuanglian Institute of Biotechnology. Cells were cultured in RPMI-1640 medium (Beijing Solarbio Science \& Technology Co., Ltd.) supplemented with 10\% FBS (Beijing Solarbio Science \& Technology Co., Ltd.) and $1 \%$ penicillin-streptomycin at $37^{\circ} \mathrm{C}$ with $5 \% \mathrm{CO}_{2}$. Cell growth was observed under a light microscope, and when cells reached $80 \%$ confluence, they were digested and passaged.

The aforementioned cells were grouped as follows: i) Control group, no treatment; ii) GA high-dose group (GA-H), cells stimulated with $60 \mu \mathrm{M}$ GA (cat no. G131992; Shanghai Aladdin Biochemical Technology Co., Ltd.) dissolved in PBS for 24 h; iii) GA medium-dose group (GA-M), cells stimulated with $30 \mu \mathrm{M}$ GA for 24 h; iv) GA low-dose group (GA-L), cells stimulated with $15 \mu \mathrm{M}$ GA for $24 \mathrm{~h}$ (19); and v) positive drug control group (5-FU), cells stimulated with $10 \mu \mathrm{M}$ 5-FU (cat. no. F0123.0005; Duchefa Biochemie) for $24 \mathrm{~h}$ (20). The experiments were performed in three parallel groups per condition.

Subsequently, to verify whether GA could inhibit SRC and EGFR phosphorylation to reduce colon cancer proliferation, cells were divided into five groups as follows: i) Control group; ii) GA-H group; iii) PP2 group, cells stimulated with $5 \mu \mathrm{M}$ SRC inhibitor PP2 (cat. no. P125361; Shanghai Aladdin Biochemical Technology Co., Ltd.) for 24 h (21); iv) gefinitib group, cells stimulated with $10 \mu \mathrm{M}$ EGFR antagonist gefinitib (Nanjing Duly Biotechnology Co. Ltd.) for $24 \mathrm{~h}$ (22); and v) GA-H + PP2 group, cells simultaneously stimulated with $60 \mu \mathrm{M} \mathrm{GA}$ and $5 \mu \mathrm{M}$ PP2 for $24 \mathrm{~h}$.

Plate clone formation assay. When HCT116 and HT29 cells reached the logarithmic growth phase, they were uniformly seeded in a six-well plate (500 cells/well) and cultured at $37^{\circ} \mathrm{C}$ with $5 \% \mathrm{CO}_{2}$ for 2-3 weeks. When the number of cells reached $50-150$, the formed colony is visible. Once formed colonies were visible, cells were fixed with $4 \%$ paraformaldehyde for $15 \mathrm{~min}$ at room temperature. Cells were subsequently stained with Giemsa (cat. no. D010-1-2; Nanjing Jiancheng Bioengineering Institute) for $25 \mathrm{~min}$ at room temperature. Following washing by PBS, the colonies were dried at room temperature for $72 \mathrm{~h}$. And image of each well was captured under x100 magnification using a light microscopy (Olympus BX51; Olympus Corporation). Finally, ImageJ v6.0 software (National Institutes of Health) was used to analyze the colony number.

Flow cytometry. After HCT116 and HT29 cells were cultured for $24 \mathrm{~h}$ in a $5 \% \mathrm{CO}_{2}$ incubator at $37^{\circ} \mathrm{C}$, the supernatant was collected and washed with PBS. A total of $2 \times 10^{5}$ cells were collected by trypsin digestion. Cells were then resuspended in $100 \mu \mathrm{l}$ binding buffer, followed by the addition of $400 \mu \mathrm{l}$ PBS. The assay was performed according to the manufacturer's instructions (cat. no. G003-1-2; Nanjing Jiancheng Bioengineering Institute). A total of $5 \mu \mathrm{l}$ PI staining solution and $10 \mu$ l Annexin V-FITC were added to each tube, and cells were incubated in the dark for $15 \mathrm{~min}$ at room temperature to detect apoptosis by Coulter ${ }^{\circledR}$ EPICS $^{\circledR} \mathrm{XL}^{\mathrm{TM}}$ flow cytometry (Beckman Coulter, Inc.). The results were analyzed using CytExpert (version 2.0; Beckman Coulter, Inc.) software.

Western blotting. Total protein from each group of HCT116 and HT29 cells or tumor tissues was extracted using RIPA buffer containing protein inhibitors (cat. no. W062-1-1; Nanjing Jiancheng Bioengineering Institute). Following centrifugation at $12,000 \mathrm{x} \mathrm{g}$ for $10 \mathrm{mins}$ at $4^{\circ} \mathrm{C}$, the protein concentration of the supernatant was quantified using a bicinchoninic acid protein assay kit (Nanjing Jiancheng Bioengineering Institute). In total, $50 \mu \mathrm{g}$ protein samples were separated by $10 \%$ SDS-PAGE and then transferred to PVDF membranes for $2 \mathrm{~h}$. After blocking with $5 \%$ non-fat dry milk at room temperature for $2 \mathrm{~h}$, primary antibodies against cleaved caspase-9 (cat. no. orb227889; 1:1,000; Biorbyt Ltd.), pro-caspase-9 (ab138412; 1:800), cleaved caspase-3 (cat.no. ab49822; 1:500), pro-caspase-3 (ab32150; 1:1,000; all from Abcam), Ki-67 (cat. no. orb475270; 1:200; Biorbyt Ltd.), rabbit anti-human phosphorylated (p)-EGFR (cat. no. ab32578; 1:1,000), EGFR (cat. no. ab52894; 1:1,000), p-AKT (cat. no. ab38449; 1:1,000), AKT (cat. no. ab8805; 1:500), p-SRC (cat. no. ab32078; $1: 5,000)$, SRC (cat. no. ab109381; 1:10,000), p-STAT3 (cat. no. ab76315; 1:2,000), STAT3 (cat. no. ab68153; 1:1,000) and $\beta$-actin (cat. no. ab8227; 1:1,000; all from Abcam) were added to the membranes and incubated overnight at $4{ }^{\circ} \mathrm{C}$. The membranes were washed with 1X TBST (containing 0.1\% Tween-20) and incubated with goat anti-rabbit horseradish peroxidase conjugated IgG secondary antibody (cat. no. ab6721; 1:2,000; Abcam) for $1 \mathrm{~h}$ at room temperature. The membranes were washed again with $1 \mathrm{X}$ TBS-T and protein bands were exposed using an ECL kit (cat. no. W028-2-1; Nanjing Jiancheng Bioengineering Institute). The relative expression of target proteins was analyzed by Image J v6.0 software (National Institutes of Health) and calculated based on $\beta$-actin as the internal reference.

Immunofluorescence. HCT116 and HT29 cells were fixed with $4 \%$ paraformaldehyde for $20 \mathrm{~min}$ at room temperature. The cells were then permeabilized with $0.5 \%$ Triton X-100 for 20 min and blocked with 5\% BSA (Wuhan Boster Biological Technology, Ltd.) for $40 \mathrm{~min}$, both at room temperature. Subsequently, the cells were incubated with p-STAT3 (cat. no. ab76315; 1:500), p-SRC (cat. no. ab32078; 1:50), p-EGFR (cat. no. ab32578; 1:25; all from Abcam) and p-AKT (cat. no. orb312153; 1:100; Biorbyt Ltd.) antibodies overnight at $4^{\circ} \mathrm{C}$. After washing with PBS, the cells were incubated with goat anti-rabbit IgG H\&L (Alexa Fluor $^{\circledR} 488$; cat. no. ab150077; 1:200; Abcam) at $37^{\circ} \mathrm{C}$ 

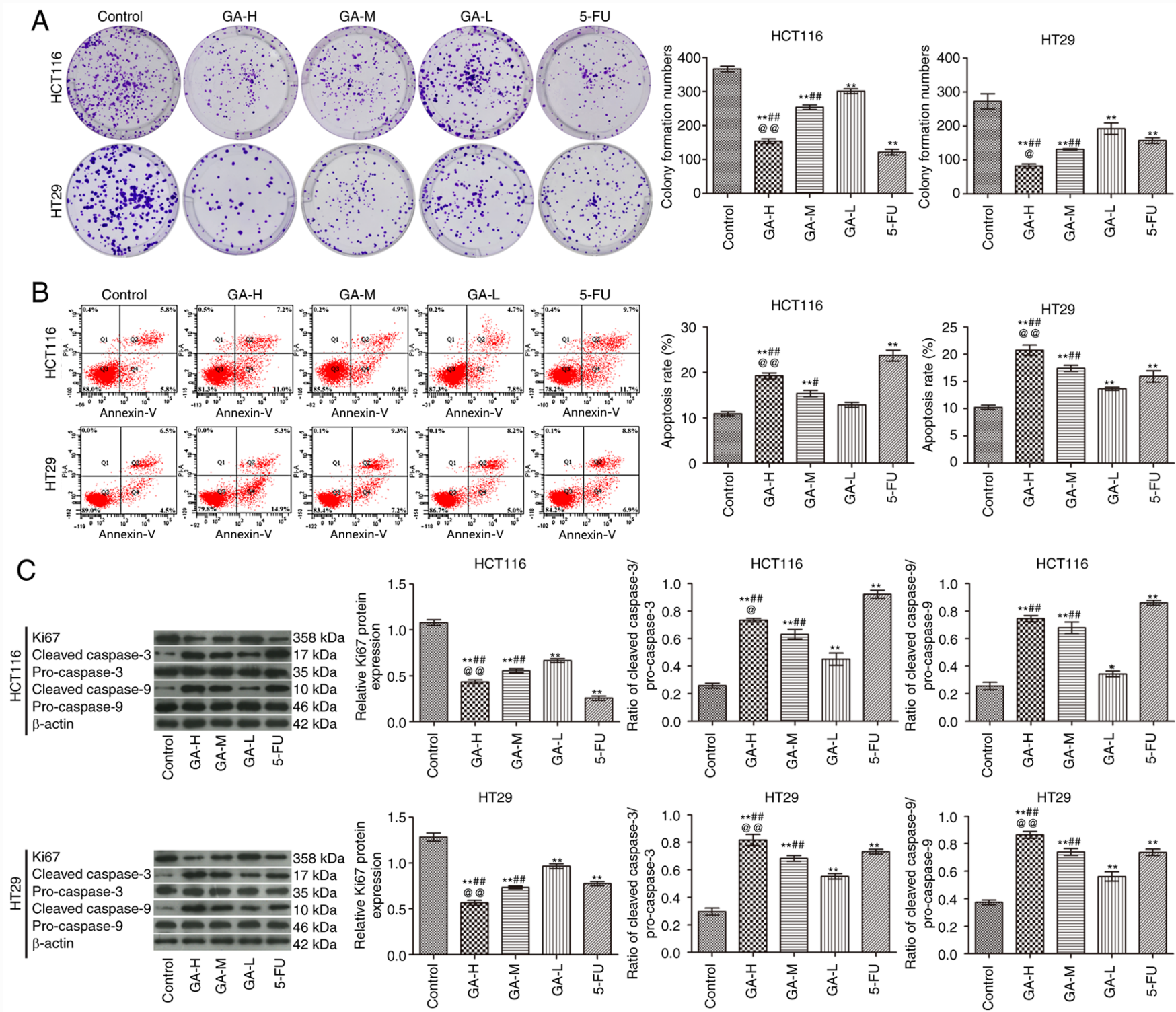

Figure 1. GA affects the apoptosis and proliferation of HCT116 and HT29 colon cancer cells. (A) Plate clone formation assay was used to examine cell proliferation. (B) Flow cytometry was used to examine apoptosis. (C) Western blotting was used to analyze the expression of proliferation-related protein Ki-67 and apoptosis-related proteins, cleaved caspase-3, pro-caspase- 3 , cleaved caspase- 9 and pro-caspase- $9(\mathrm{n}=3)$. ${ }^{*} \mathrm{P}<0.05$ and ${ }^{* *} \mathrm{P}<0.01$ vs. the control group; ${ }^{*} \mathrm{P}<0.05$ and ${ }^{\# \#} \mathrm{P}<0.01$ vs. the GA-L group; ${ }^{\circledR} \mathrm{P}<0.05$ and ${ }^{\circledR}{ }^{\circledR} \mathrm{P}<0.01$ vs. the GA-M group. GA, gallic acid; L, low dose; $\mathrm{M}$, medium dose; H, high dose; 5-FU, 5-fluorouracil.

for $1.5 \mathrm{~h}$. Following three washes with PBS, the nuclei were stained with $5 \mu \mathrm{g} / \mathrm{ml}$ DAPI for $5 \mathrm{~min}$ at room temperature. Immunofluorescence was observed by confocal microscopy (Nikon Corporation) with the magnification of $x 400$ and the mean fluorescence intensity were quantified using Image J v6.0 software (National Institutes of Health).

Animals. A total of 48 specific-pathogen-free BALB/c female nude mice (16-18 g; 4 weeks old) were purchased from Beijing Weitong Lihua Laboratory Animal Technology Co., Ltd. [SCXK (Beijing) 20160006]. Operations were performed after 7 days of adaptive feeding. Animal experiments in the present study were agreed and approved by the Animal Protection and Use Committee of Yantai University (Yantai, China). The animals were raised at $26-28^{\circ} \mathrm{C}, 40-60 \%$ humidity, light/dark cycle for $12-\mathrm{h}$ and free access to water and food. The food, drinking water and bedding materials were sterilized. Animal experiments followed the National Institutes of Health guidelines (NIH publication no. 85-23, revised 1996).

Xenograft tumor model. HCT116 and HT29 cells were suspended with RPMI-1640 medium without 10\% FBS. A total of $0.2 \mathrm{ml} \mathrm{HCT116}$ and HT29 cell suspended with PBS $\left(5 \times 10^{7}\right.$ cells $\left./ \mathrm{ml}\right)$ were inoculated subcutaneously into the left backs of nude mice in the corresponding group. If a rice-sized tumor $\left(\sim 50 \mathrm{~mm}^{3}\right)$ appeared at the inoculation site on the 7 th day, the inoculation was considered to be successful. Nude mice were randomly divided into four groups $(n=6)$ : i) Model, mice inoculated with cancer cells; ii) GA group, following inoculation of cancer cells, mice were injected daily with $80 \mathrm{mg} / \mathrm{kg} /$ day GA (23); iii) PP2 group, following inoculation of cancer cells, mice were injected daily with $10 \mathrm{mg} / \mathrm{kg} /$ day PP2 (24); iv) gefitinib group, following inoculation of cancer cells, mice were injected daily with $50 \mathrm{mg} / \mathrm{kg} /$ day gefitinib (25); and v) GA + 

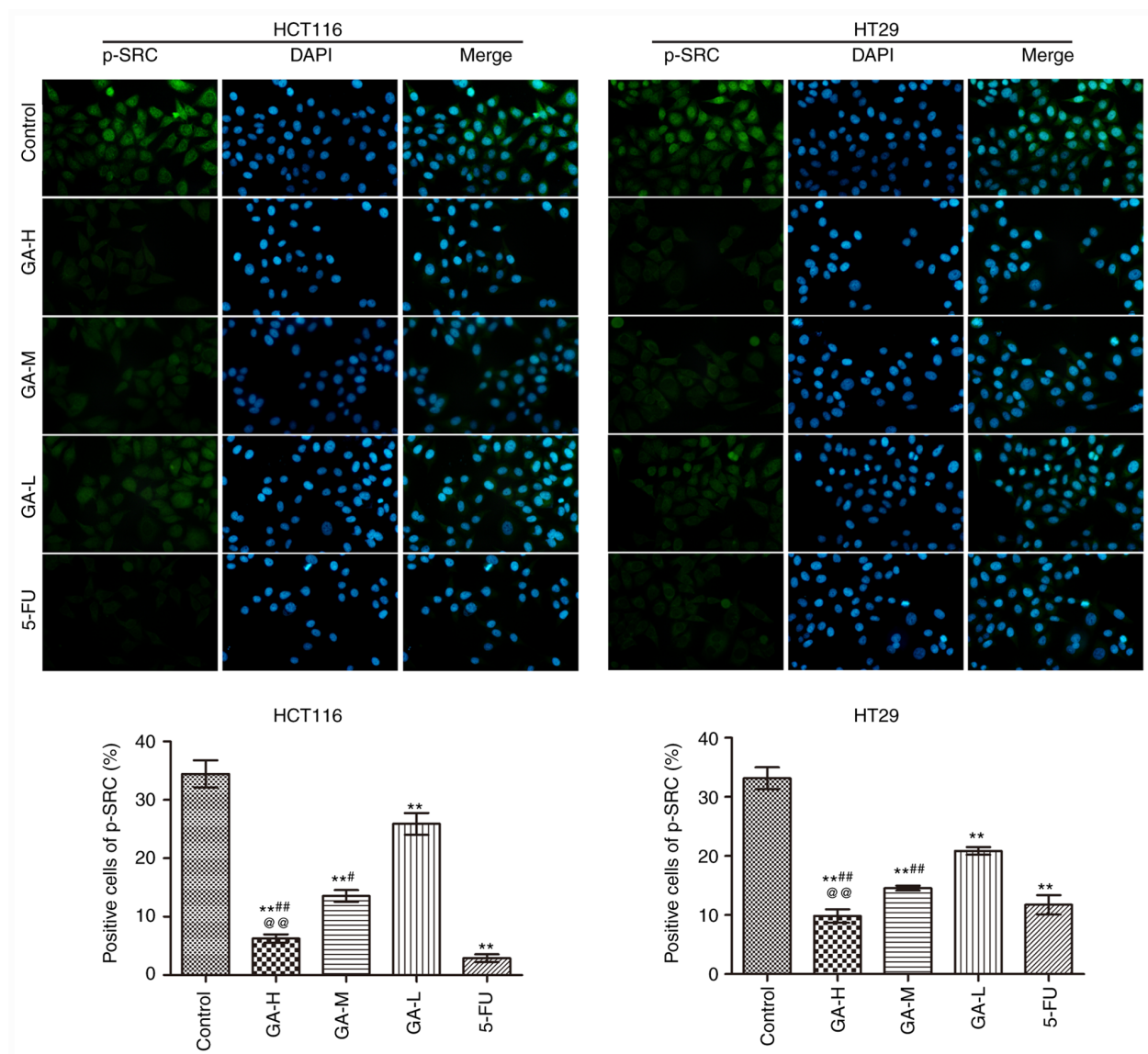

Figure 2. GA inhibits p-SRC expression in HCT116 and HT29 cells by Immunofluorescence ( $\mathrm{n}=3$; magnification, $\mathrm{x} 400)$. ${ }^{* *} \mathrm{P}<0.01$ vs. the control group; ${ }^{*} \mathrm{P}<0.05$ and ${ }^{\# /} \mathrm{P}<0.01$ vs. the GA-L group; ${ }^{\circledR @} \mathrm{P}<0.01$ vs. the GA-M group. GA, gallic acid; L, low dose; M, medium dose; H, high dose; 5-FU, 5-fluorouracil; p, phosphorylated.

PP2 group, following inoculation of cancer cells, mice were injected daily with $80 \mathrm{mg} / \mathrm{kg} / \mathrm{day}$ GA and $10 \mathrm{mg} / \mathrm{kg} / \mathrm{day} \mathrm{PP} 2$. Continuous administration was performed for 28 days. Tumor volumes were recorded every 7 days. At the end of the experiment, the mice were anesthetized by intraperitoneal injection of $3 \%$ sodium pentobarbital $(40 \mathrm{mg} / \mathrm{kg})$ and sacrificed by cervical dislocation. Death was confirmed by cardiac arrest. Tumors were excised, photographed and weighed.

TUNEL assay. Tumor tissues were fixed with $4 \%$ paraformaldehyde for $24 \mathrm{~h}$ at room temperature, dehydrated and embedded in paraffin. The slices $(5 \mu \mathrm{m})$ were heated at $60-70^{\circ} \mathrm{C}$ for $2 \mathrm{~h}$, dewaxed with xylene and then rehydrated using a descending ethanol gradient. The tissue slices were incubated in $3 \% \mathrm{H}_{2} \mathrm{O}_{2}$-methanol for $10 \mathrm{~min}$ at room temperature and rinsed with PBS three times ( 3 min each time). Subsequently, the slices were stained with TUNEL solution (cat. no. G002-2-2; Nanjing Jiancheng Bioengineering Institute). A sealing film was used to perform the reaction in a dark wet box for $1 \mathrm{~h}$ at $37^{\circ} \mathrm{C}$. After TUNEL-staining, DAB solution was added for $5 \mathrm{~min}$ at room temperature. After washing with PBS for three times, the slices were re-stained using hematoxylin at room temperature for $60 \mathrm{sec}$, then dehydrated in ascending series of ethanol and cleared in xylene. Finally, the slices were sealed with neutral gum and observed under a light microscope (magnification, x400; Olympus BX51; Olympus Corporation) from five randomly selected fields. The number of positive-stained cells was analyzed by ImageJ v6.0 software (National Institutes of Health). Cell apoptosis rate was calculated using the following formula: Apoptosis rate $=$ (number of apoptotic cells/total number of cells) $\times 100 \%$.

Statistical analysis. All data were analyzed with SPSS v19.0 (IBM Corp.). Numerical data are presented as the mean \pm SD. Differences among multiple groups were evaluated by one-way ANOVA followed by Tukey's post hoc test. $\mathrm{P}<0.05$ was considered to indicate a statistically significant difference.

\section{Results}

GA promotes apoptosis and inhibits colon cancer cell proliferation. Compared with the control group, GA inhibited the proliferation (Fig. 1A) and promoted the apoptosis (Fig. 1B) of HCT116 and HT29 cells in a concentration-dependent manner. In addition, GA increased the ratio of cleaved caspase-3/pro-caspase-3 and cleaved caspase-9/pro-caspase-9 and inhibited $\mathrm{Ki}-67$ expression when compared with the control group (Fig. 1C). Notably, the effects of GA-H treatment were similar with those observed with 5-FU treatment. These data demonstrated that GA administration promoted apoptosis and inhibited proliferation in colon cancer cells.

GA inhibits the phosphorylation of SRC in colon cancer cells. The effects of GA on SRC phosphorylation in HCT116 and HT29 cells were analyzed using immunofluorescence (Fig. 2). GA and 5-FU intervention significantly reduced the positive p-SRC staining in HCT116 and HT29 cells compared with 
A
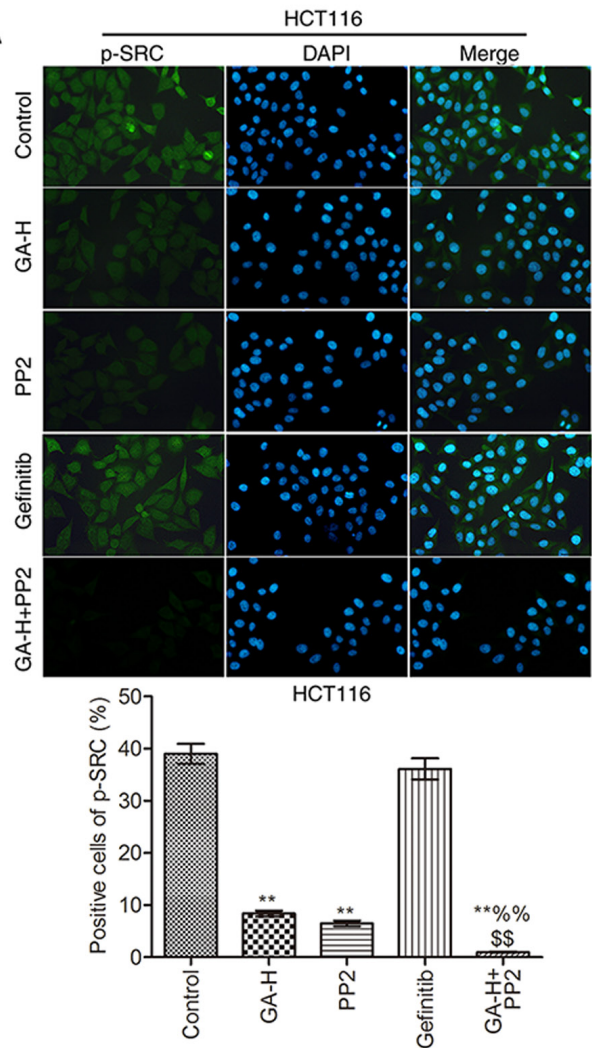

B

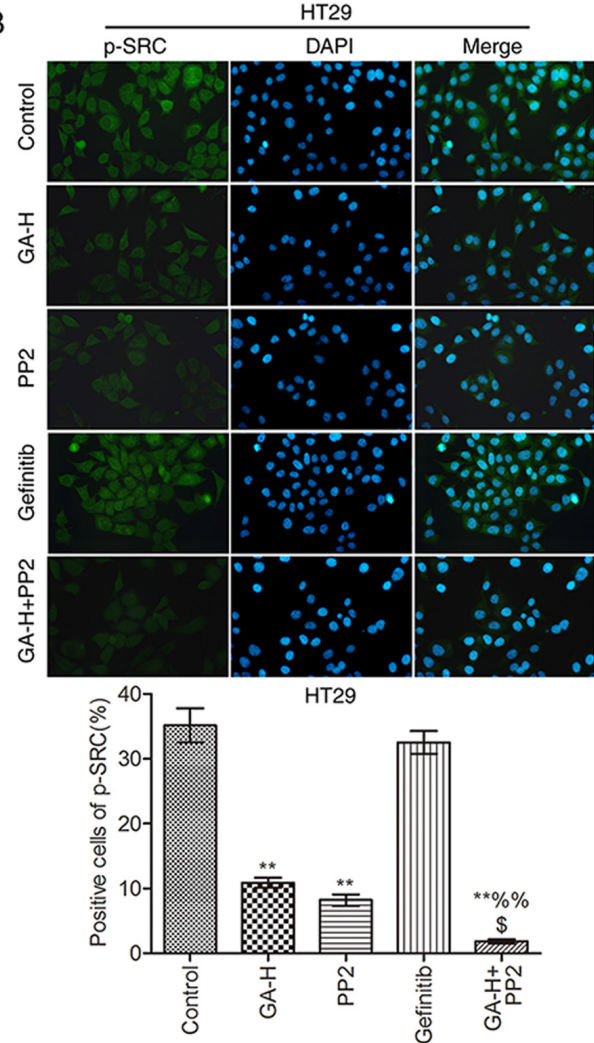

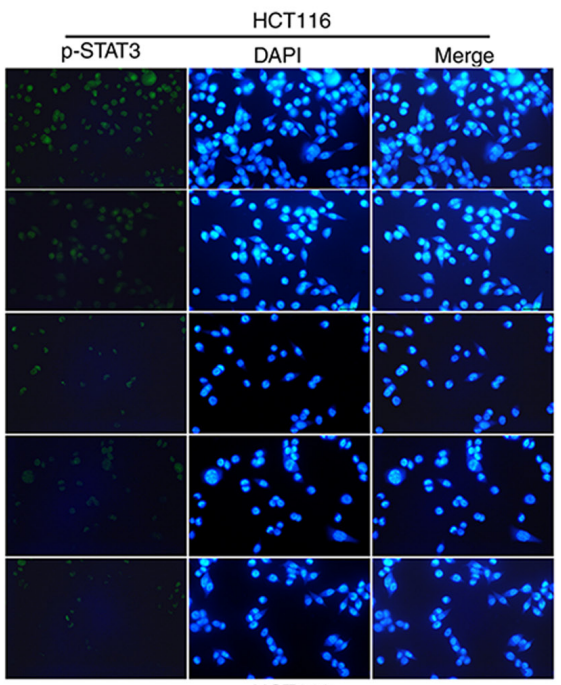
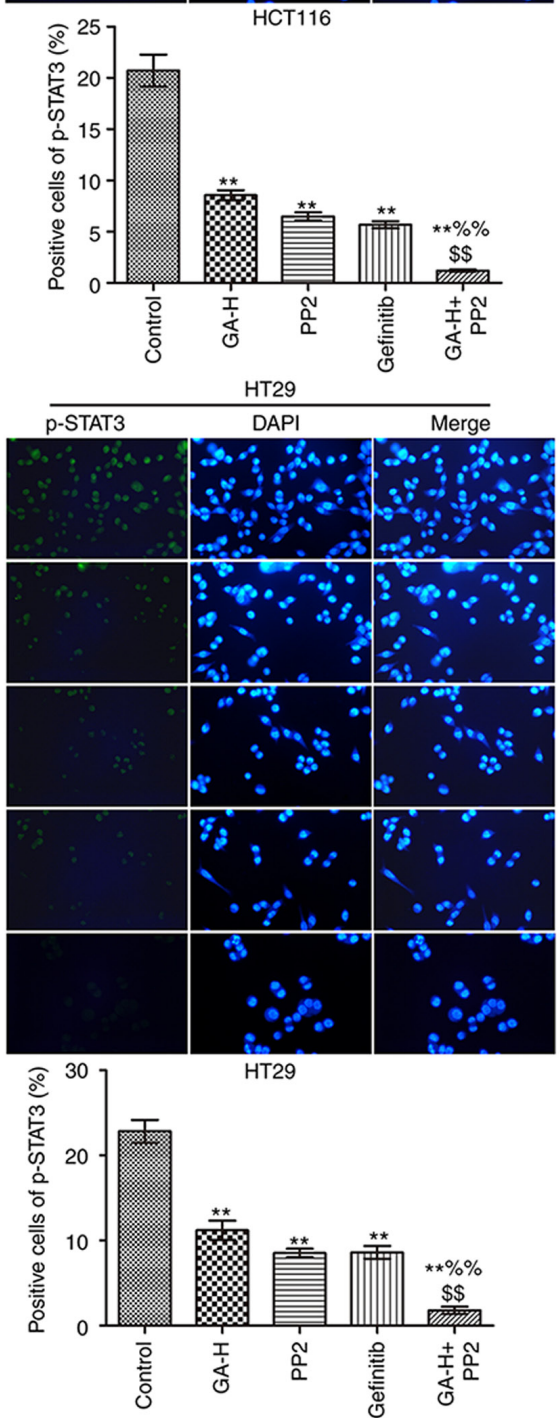

Figure 3. Effects of GA on p-SRC and p-STAT3 level. The effects of GA in (A) HCT116 and (B) HT29 colon cancer cells were analyzed by immunofluorescence. $\mathrm{n}=3$. Magnification, $\mathrm{x} 400 .{ }^{* *} \mathrm{P}<0.01$ vs. the control group; ${ }^{\%} \% \mathrm{P}<0.01$ vs. the GA-H group; ${ }^{\$} \mathrm{P}<0.05$ and ${ }^{\$ \$} \mathrm{P}<0.01 \mathrm{vs}$. the PP2 group. GA-H, gallic acid-high dose; p, phosphorylated.

the control group, especially in 5-FU. Moreover, high GA concentrations exerted the strongest inhibitory effect on SRC phosphorylation in HCT116 cells and HT29 cells. These data suggested that GA suppressed SRC phosphorylation.
GA decreases STAT3 and AKT phosphorylation level via inhibiting SRC and EGFR phosphorylation. The levels of p-SRC, p-STAT3 (Fig. 3), p-EGFR and p-AKT (Fig. 4) were examined using immunofluorescence. Compared with the 

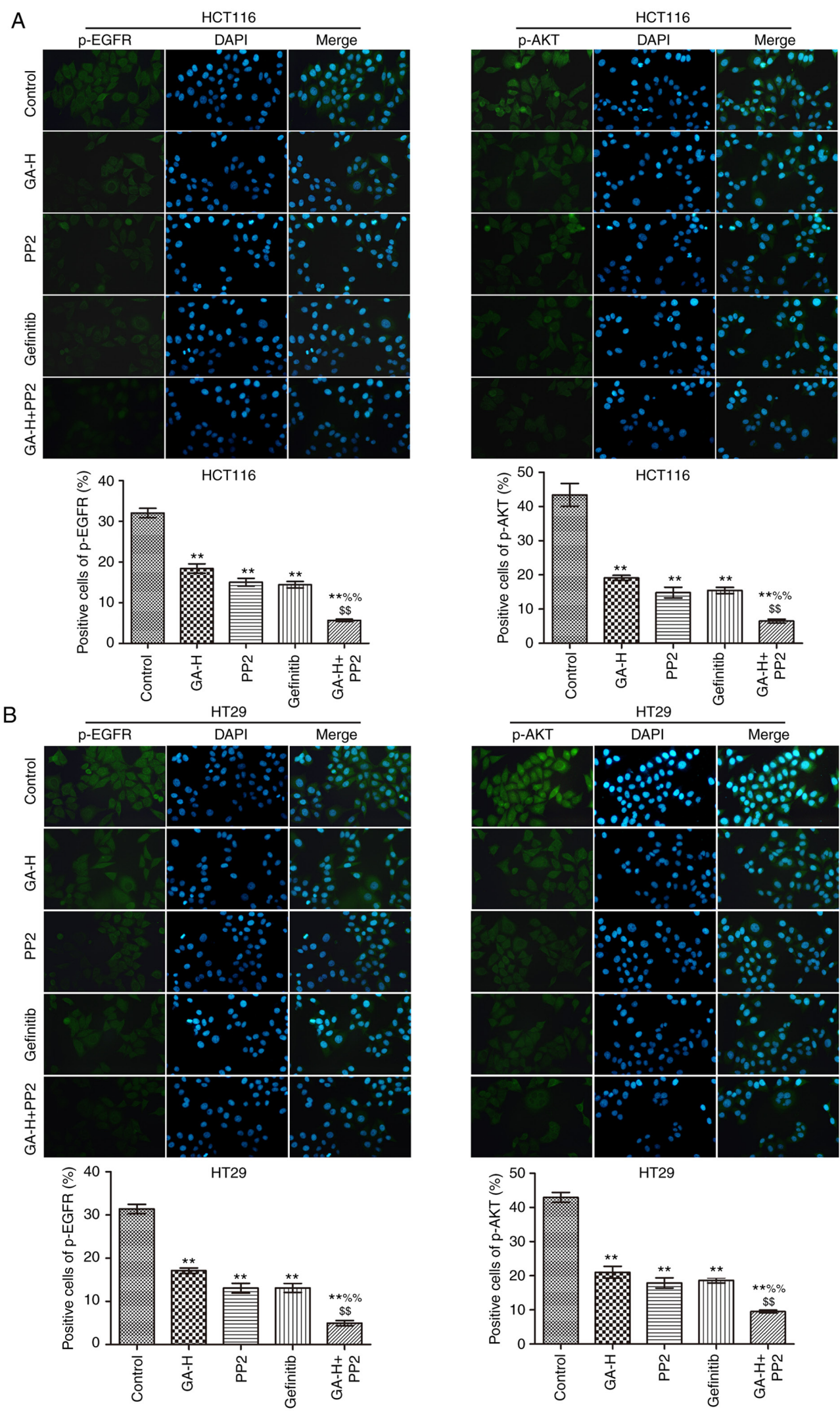

Figure 4. Effects of GA on p-EGFR and p-AKT level. The effects of GA in (A) HCT116 and (B) HT29 colon cancer cells were analyzed by immunofluorescence. $\mathrm{n}=3$. Magnification, $\mathrm{x} 400 .{ }^{* * *} \mathrm{P}<0.01$ vs. the control group; ${ }^{\% \%} \mathrm{P}<0.01$ vs. the GA-H group; ${ }^{\$ \$} \mathrm{P}<0.01$ vs. the PP2 group. GA-H, gallic acid-high dose; p, phosphorylated.

control group, GA-H and PP2 markedly inhibited the level of p-STAT3, p-EGFR, p-AKT and p-SRC, and gefitinib significantly reduced the level of p-STAT3, p-EGFR and
p-AKT, but had no effect on p-SRC level. When GA-H and PP2 were used in combination, the phosphorylation level of the aforementioned proteins was further decreased compared 

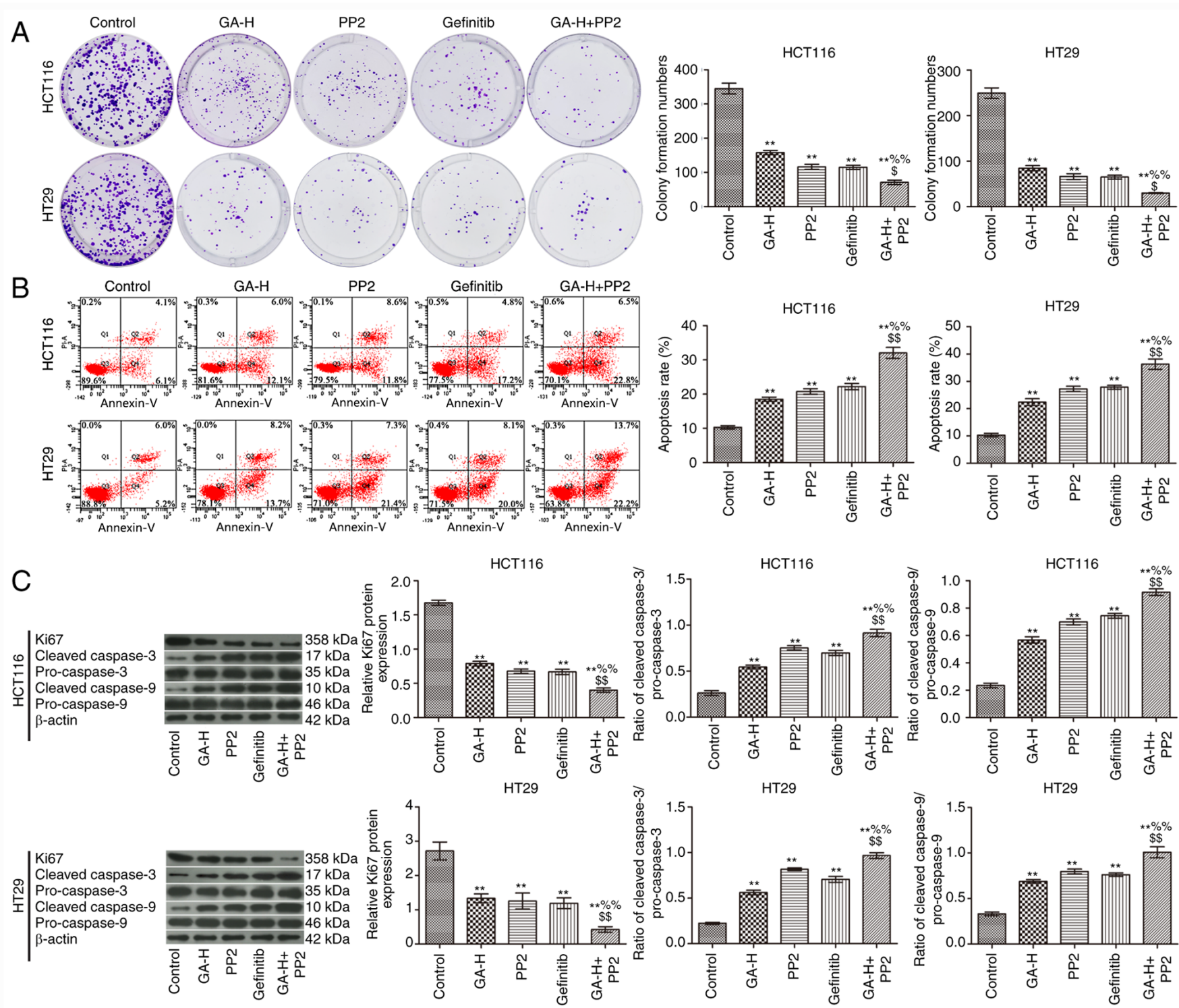

Figure 5. GA decreases SRC and EGFR phosphorylation to promote apoptosis and inhibit proliferation in HCT116 and HT29 cells. (A) Plate clone formation assay was used to examine cell proliferation. (B) Flow cytometry was used to examine apoptosis. (C) Western blotting was used to analyze the expression of proliferation-related protein $\mathrm{Ki}-67$ and apoptosis-related proteins, cleaved caspase-3, pro-caspase- 3 , cleaved caspase- 9 and pro-caspase- 9 . $\mathrm{n}=3$. ${ }^{* * *} \mathrm{P}<0.01 \mathrm{vs.}$. the control group; ${ }^{\% \%} \mathrm{P}<0.01$ vs. the GA-H group; ${ }^{\$} \mathrm{P}<0.05$ and ${ }^{\$ \$} \mathrm{P}<0.01$ vs. the $\mathrm{PP} 2$ group. GA-H, gallic acid-high dose.

with the GA-H or PP2 groups alone. These results indicated that GA could decrease SRC and EGFR phosphorylation level to inhibit STAT3 and AKT phosphorylation.

GA decreases SRC and EGFR phosphorylation level to promote colon cancer cell apoptosis and inhibit proliferation. To verify whether GA could inhibit SRC and EGFR phosphorylation to reduce colon cancer cell proliferation, cells were also treated with PP2 and gefitinib. As indicated in Fig. 5, GA-H, PP2 and gefitinib significantly inhibited cell proliferation (Fig. 5A) and promoted cell apoptosis (Fig. 5B) compared with the control group. The combined administration of GA-H and PP2 further promoted cell apoptosis and inhibited proliferation compared with the GA-H or PP2 groups. In addition, western blot analysis demonstrated that GA-H, PP2 and gefitinib increased the ratio of cleaved caspase-3/pro-caspase-3 and cleaved caspase-9/pro-caspase-9, and decreased Ki-67 protein expression level compared with the control group. The combined administration of GA-H and PP2 further enhanced the ratio of cleaved caspase-3/pro-caspase- 3 and cleaved caspase-9/pro-caspase- 9 , and reduced $\mathrm{Ki}-67$ protein expression level compared with the GA-H or PP2 groups (Fig. 5C). These results revealed that GA promoted colon cancer cell apoptosis and inhibited proliferation via inhibiting SRC and EGFR phosphorylation.

GA decreases SRC and EGFR phosphorylation level to inhibit tumor growth. In the present study, the maximum tumor diameter was $1.56 \mathrm{~cm}$, and the maximum tumor volume was $1,900.4 \mathrm{~mm}^{3}$. GA-H, PP2 and gefitinib inhibited tumor growth compared with the model group (Fig. 6A). When GA-H and PP2 were administered in combination, tumor growth was significantly reduced compared with the GA or PP2 groups alone. The results in Fig. 6B demonstrated 
A
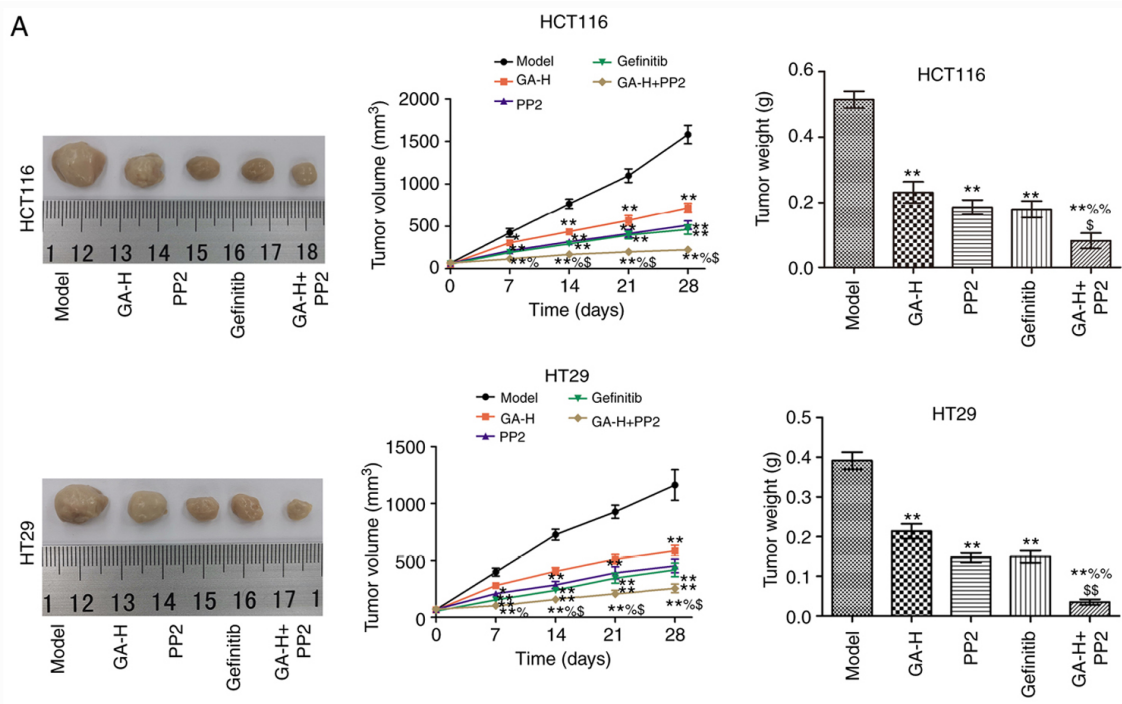

B
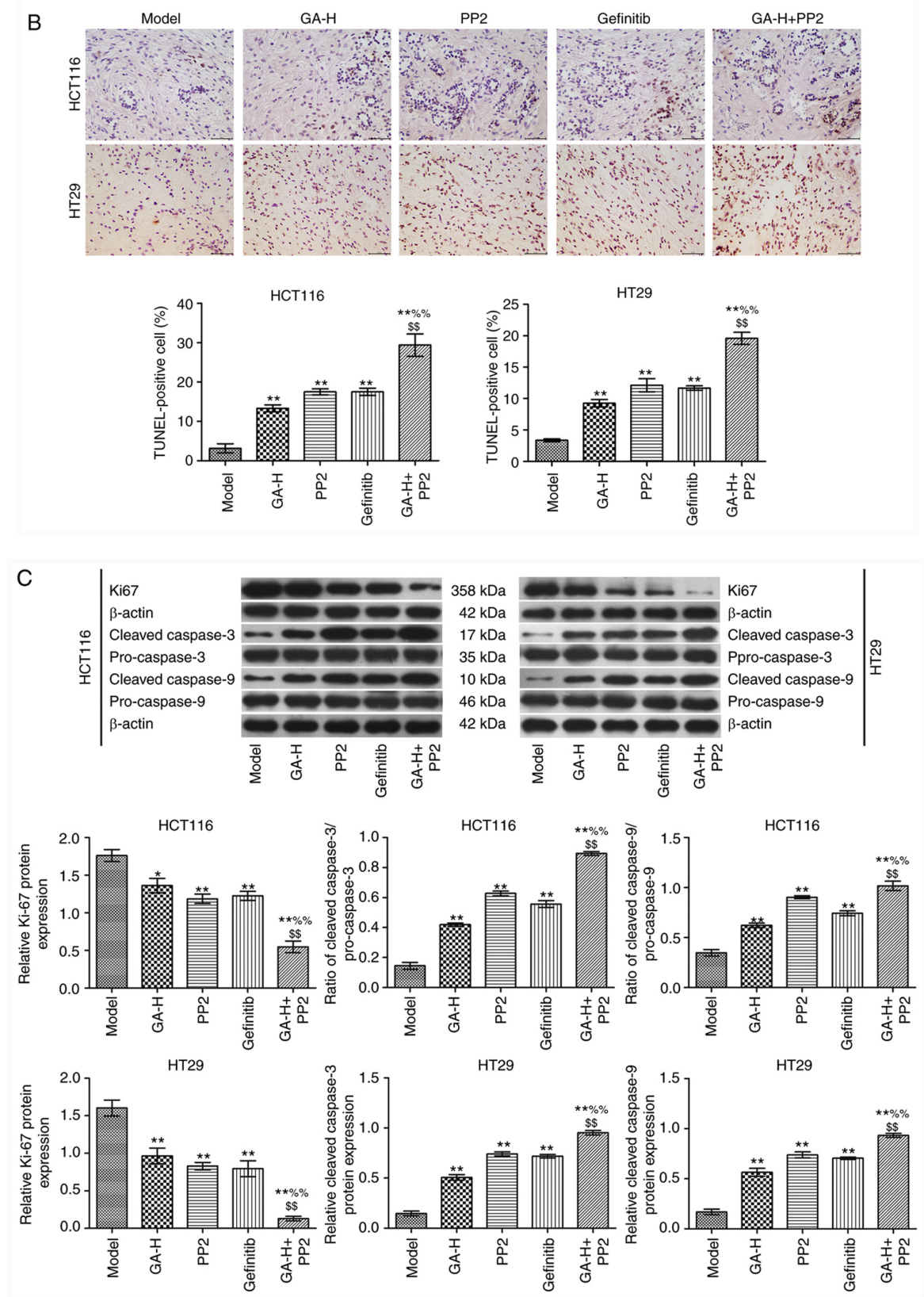

Figure 6. GA decreases SRC and EGFR phosphorylation level to inhibit tumor growth. (A) Tumor images, volume and weight. (B) TUNEL staining (scale bar $=50 \mu \mathrm{m}$ ). (C) Western blot analysis. $\mathrm{n}=6 .{ }^{*} \mathrm{P}<0.05$ and ${ }^{* *} \mathrm{P}<0.01$ vs. the model group; ${ }^{\%} \mathrm{P}<0.05$ and ${ }^{\% \%}{ }^{\circ} \mathrm{P}<0.01$ vs. the GA-H group; ${ }^{{ }^{~} \mathrm{P}<0.05}$ and ${ }^{\$ \$} \mathrm{P}<0.01$ vs the PP2 group. GA-H, gallic acid-high dose. 
A

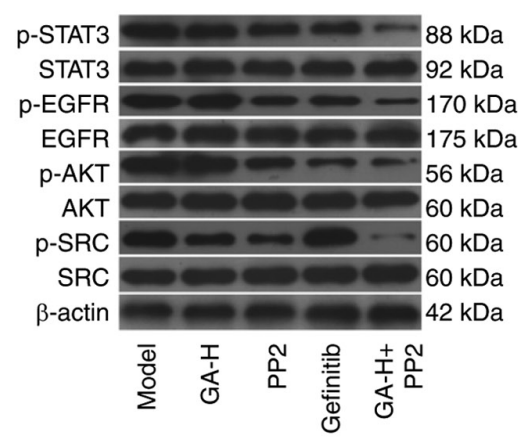

B

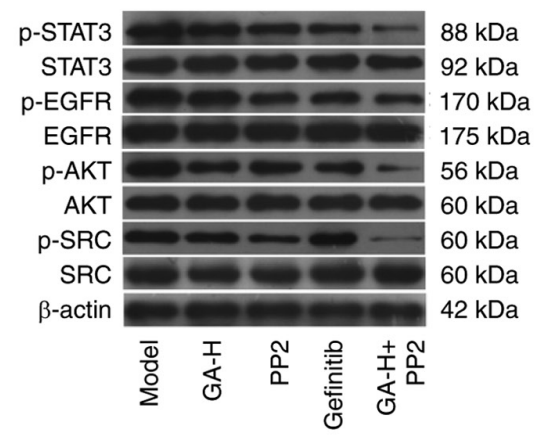

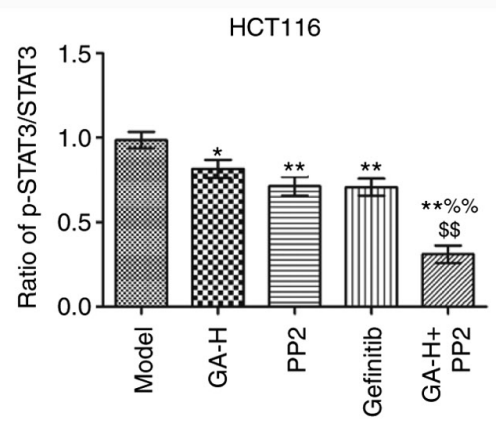

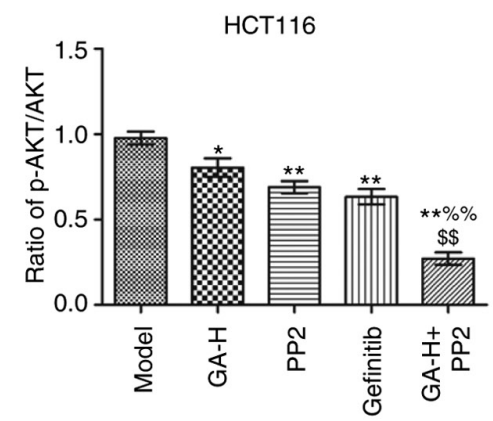

HT29

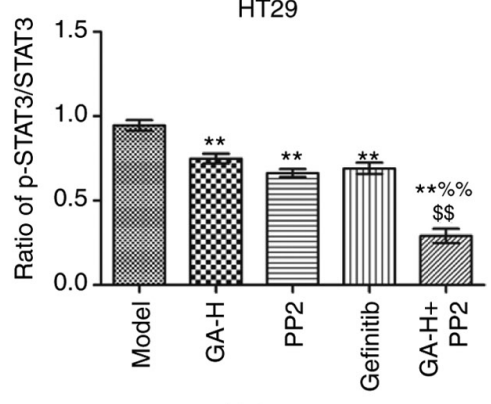

HT29

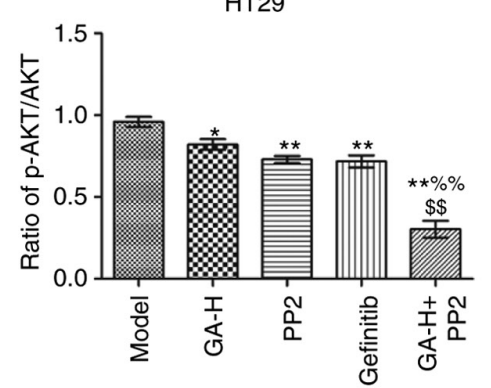

HCT116

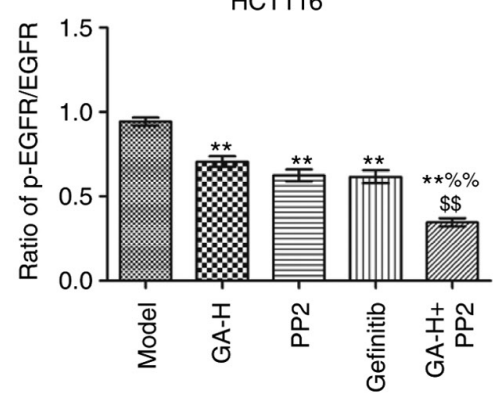

HCT116

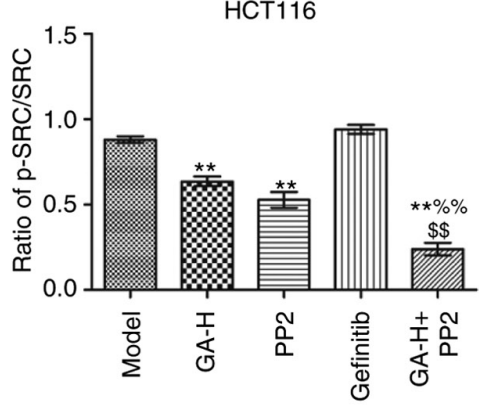

HT29

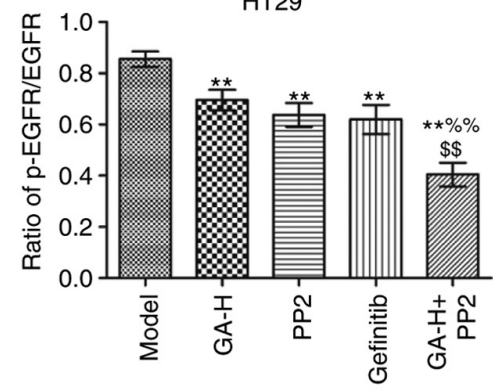

HT29

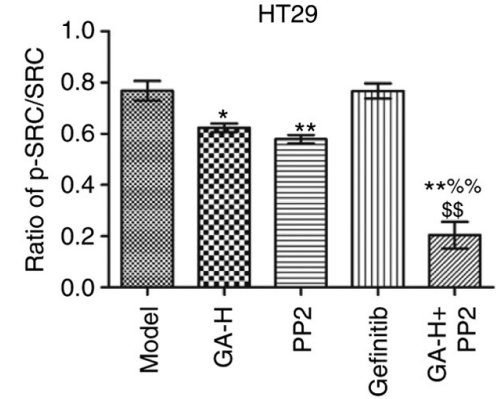

Figure 7. GA decreases SRC and EGFR phosphorylation level to inhibit STAT3 and AKT phosphorylation. (A) The proteins expression in HCT116 tumor tissues. (B) The proteins expression in HT29 tumor tissues. The level of p-SRC/SRC, p-EGFR/EGFR, p-STAT3/STAT3 and p-AKT/AKT in tumor tissues were analyzed by western blotting. $\beta$-actin was used as the internal reference gene. $\mathrm{n}=6$. ${ }^{*} \mathrm{P}<0.05$ and ${ }^{* * *} \mathrm{P}<0.01 \mathrm{vs}$. the control group; ${ }^{\% \%} \mathrm{P}<0.01 \mathrm{vs}$. the GA-H group; ${ }^{\$} \mathrm{P}<0.01$ vs. the PP2 group. GA-H, gallic acid-high dose; $p$, phosphorylated.

that GA-H, PP2 and gefitinib significantly increased the apoptosis rate of tumor cells in vivo compared with the model group. This effect was enhanced in the GA-H + PP2 group compared with the GA or PP2 groups. The expression level of Ki67 and the ratio of cleaved caspase-3/pro-caspase-3 and cleaved caspase-9/pro-caspase-9 are presented in Fig. 6C. The results revealed that GA-H, PP2 and gefitinib decreased $\mathrm{Ki}-67$ expression level and increased the ratio of cleaved caspase-3/pro-caspase-3 and cleaved caspase-9/pro-caspase- 9 compared with the model group. These effects were further enhanced when GA-H and PP2 were administered concurrently.
GA inhibits SRC and EGFR phosphorylation to regulate STAT3 and AKT in vivo. The relative levels of p-STAT3/STAT3, p-EGFR/EGFR, p-AKT/AKT and p-SRC/SRC in tumor tissues was analyzed by western blotting (Fig. 7). The results indicated that the administration of GA-H and PP2 significantly decreased the ratio of $\mathrm{p}-\mathrm{STAT} 3 / \mathrm{STAT} 3, \mathrm{p}-\mathrm{EGFR} / \mathrm{EGFR}$, p-AKT/AKT and p-SRC/SRC in tumor tissues compared with the model group (Fig. 7). Gefitinib significantly reduced the level of p-STAT3/STAT3, p-EGFR/EGFR and p-AKT/AKT, but did not affect $\mathrm{p}$-SRC level compared with the model group. Furthermore, following the combined treatment of GA-H and PP2, the phosphorylation level of the aforementioned four 
proteins was significantly lower compared with the GA-H or PP2 groups.

\section{Discussion}

The present study demonstrated that GA significantly suppressed the proliferation of HCT116 and HT29 colon cancer cells and accelerated their apoptotic process, which was consistent with the results of previous studies. In previous studies, GA has been indicated to inhibit cell proliferation and invasion of different tumors, such as non-small cell lung cancer (NSCLC) $(26,27)$ and bladder cancer $(28)$. The occurrence of colon cancer is closely associated with the abnormal expression of multiple genes (29). Therefore, regulating the expression of certain key genes, such as SRC, during tumorigenesis to inhibit malignant transformation is an effective means to control tumor growth.

The SRC protein is encoded by the proto-oncogene $\mathrm{Src}$ and is a member of the non-receptor tyrosine protein kinase family, which can activate multiple signaling pathways, such as PI3K/AKT, MAPK and STAT3 (30). SRC activity is enhanced in $80 \%$ of patients with colon cancer, and is significantly associated with the occurrence and development of colon cancer $(31,32)$. In the present study, GA was administered to HCT116 and HT29 colon cancer cells and colon cancer animal models. It was revealed that GA treatment significantly inhibited the proliferation and promoted the apoptosis of colon cancer cells, and this effect was associated with p-SRC level.

The EGFR signal transduction pathway is also widely considered to serve a key role in tumor formation and development and is one of the most important targets for numerous cancers $(33,34)$. It often exhibits abnormal activation during cancer progression and is closely associated with poor prognosis (33). Nam et al (34) demonstrated that GA induced apoptosis in EGFR-mutant NSCLC via acceleration of EGFR turnover, which suggested that GA-mediated inhibition of cancer growth may be associated with EGFR signaling. Previously, SRC was indicated to activate EGFR via different pathways to promote tumor proliferation and metastasis (35). In addition, STAT3 has been also revealed to be continuously activated in tumors, which serves crucial roles in regulating the proliferation, migration and survival of cells and can be also regulated by SRC (36). In present study, GA was indicated to inhibit SRC and EGFR phosphorylation resulting in a decrease in STAT3 and AKT phosphorylation level. When the SRC inhibitor PP2 and the EGFR inhibitor gefitinib were administered in vitro or in vivo, these results were further confirmed. However, Chen et al (37) demonstrated that constitutively active AKT and MEK-1 or SRC overexpression reversed the inhibitory effect of GA on the EGF-induced MMP-9 upregulation in breast cancer cells. It is possible that the reported effects are the results of the effect of GA on different, independent pathways and not part of the mechanism mediating the effect on tumor growth.

Few studies have investigated the mechanism of GA in colon cancer in vivo and in vitro. The present study demonstrated that GA inhibited colon cancer cell proliferation and induced apoptosis via inhibiting SRC and EGFR phosphorylation. The results of the present study may provide novel ideas, strategies and treatment options for the prevention and targeted therapy of colon cancer.

\section{Acknowledgements}

Not applicable.

\section{Funding}

No funding was received.

\section{Availability of data and materials}

The datasets used and/or analyzed during the current study are available from the corresponding author on reasonable request.

\section{Authors' contributions}

XL, LH and PL conceived the study and performed literature research, data analysis, experiments, manuscript writing and review; GW and YG designed the experiments and were involved in the data acquisition and statistical analysis; TW was involved in data acquisition, manuscript preparation and data analysis; KC performed study design, literature research, experiments and manuscript editing. XL and GW confirmed the authenticity of the raw data. All authors read and approved the final manuscript.

\section{Ethics approval and consent to participate}

Animal experiments followed the NIH guidelines (NIH publication no. 85-23, revised 1996) and were approved by the Animal Protection and Use Committee of Yantai University (Yantai, China).

\section{Patient consent for publication}

Not applicable.

\section{Competing interests}

The authors declare that they have no competing interests.

\section{References}

1. Arnold M, Sierra MS, Laversanne M, Soerjomataram I, Jemal A and Bray F: Global patterns and trends in colorectal cancer incidence and mortality. Gut 66: 683-691, 2017.

2. Favoriti P, Carbone G, Greco M, Pirozzi F, Pirozzi RE and Corcione F: Worldwide burden of colorectal cancer: A review. Updates Surg 68: 7-11, 2016.

3. Giftson JS, Jayanthi S and Nalini N: Chemopreventive efficacy of gallic acid, an antioxidant and anticarcinogenic polyphenol, against 1,2-dimethyl hydrazine induced rat colon carcinogenesis. Invest New Drugs 28: 251-259, 2010

4. Carole M,Livine D, Daniel K, Järvinen JR, Annelise L and Muller CD: Impact of procyanidins from different berries on caspase 8 activation in colon cancer. Oxid Med Cell Longev 2015: 154164, 2015.

5. Grothey A and Venook AP: Optimizing adjuvant therapy for localized colon cancer and treatment selection in advanced colorectal cancer. J Natl Compr Canc Netw 16: 611-615, 2018.

6. No authors listed: Adjuvant chemotherapy with oxaliplatin, in combination with fluorouracil plus leucovorin prolongs disease-free survival, but causes more adverse event cancer Abstracted from: Andre T, Boni C, Mounedji-Boudiaf L, et al. Multicenter international study of oxaliplatin/5-fluorouracil/leucovorin in the adjuvant treatment of colon cancer (MOSAIC) investigators. Oxaliplatin, fluorouracil, and leucovorin as adjuvant treatment for colon cancer. N Engl J Med 350: 2343-2351, 2004. Cancer Treat Rev 30: 711-713, 2004. 
7. Schmoll HJ, Tabernero J, Maroun J, de Braud F, Price T, Van Cutsem E, Hill M, Hoersch S, Rittweger K and Haller DG: Capecitabine plus oxaliplatin compared with fluorouracil/folinic acid as adjuvant therapy for stage III colon cancer: Final results of the NO16968 randomized controlled phase III trial. J Clin Oncol 33: 3733-3740, 2015.

8. Liu X, Jiang J, Chan R, Ji Y, Lu J, Liao YP, Okene M, Lin J, Lin P, Chang $\mathrm{CH}$, et al: Improved efficacy and reduced toxicity using a custom-designed irinotecan-delivering silicasome for orthotopic colon cancer. ACS Nano 13: 38-53, 2019.

9. Deng S, Shanmugam MK, Kumar AP, Yap CT, Sethi G and Bishayee A: Targeting autophagy using natural compounds for cancer prevention and therapy. Cancer 125: 1228-1246, 2019.

10. Yaffe PB, Power Coombs MR, Doucette CD, Walsh M and Hoskin DW: Piperine, an alkaloid from black pepper, inhibits growth of human colon cancer cells via G1 arrest and apoptosis triggered by endoplasmic reticulum stress. Mol Carcinog 54 1070-1085, 2015

11. Venancio VP, Cipriano PA, Kim H, Antunes LM, Talcott ST and Mertens-Talcott SU: Cocoplum (Chrysobalanus icaco L.) anthocyanins exert anti-inflammatory activity in human colon cancer and non-malignant colon cells. Food Funct 8: 307-314, 2017.

12. Kubow S, Iskandar MM, Melgar-Bermudez E, Sleno L, Sabally K, Azadi B, How E, Prakash S, Burgos G and Felde TZ: Effects of simulated human gastrointestinal digestion of two purple-fleshed potato cultivars on anthocyanin composition and cytotoxicity in colonic cancer and non-tumorigenic cells Nutrients 9: 953, 2017.

13. Mazewski C, Liang K and de Mejia EG: Inhibitory potential of anthocyanin-rich purple and red corn extracts on human colorectal cancer cell proliferation in vitro. J Funct Foods 34 254-265, 2017.

14. Niemetz R and Gross GG: Enzymology of gallotannin and ellagitannin biosynthesis. Phytochemistry 66: 2001-2011, 2005.

15. Kroes BH, van den Berg AJ, Quarles van Ufford HC, van Dijk H and Labadie RP: Anti-inflammatory activity of gallic acid. Planta Med 58: 499-504, 1992.

16. Choi HJ, Song JH, Bhatt LR and Baek SH: Anti-human rhinovirus activity of gallic acid possessing antioxidant capacity. Phytother Res 24: 1292-1296, 2010.

17. You BR and Park WH: Gallic acid-induced lung cancer cell death is related to glutathione depletion as well as reactive oxygen species increase. Toxicol In Vitro 24: 1356-1362, 2010.

18. Sun GL and Wang D: Gallic acid from terminalia chebula inhibited the growth of esophageal carcinoma cells by suppressing the Hippo signal pathway. Iran J Basic Med Sci 23: 1401-1408, 2020

19. Lee HL, Lin CS, Kao SH and Chou MC: Gallic acid induces G1 phase arrest and apoptosis of triple-negative breast cancer cell MDA-MB-231 via p38 mitogen-activated protein kinase/p21/p27 axis. Anticancer Drugs 28: 1150-1156, 2017.

20. Li J, Hou N, Faried A, Tsutsumi S, Takeuchi T and Kuwano H: Inhibition of autophagy by 3-MA enhances the effect of 5-FU-induced apoptosis in colon cancer cells. Ann Surg Oncol 16: 761-771, 2009.

21. Kundu J, Choi BY, Jeong CH, Kundu JK and Chun KS: Thymoquinone induces apoptosis in human colon cancer HCT116 cells through inactivation of STAT3 by blocking JAK2and Src-mediated phosphorylation of EGF receptor tyrosine kinase. Oncol Rep 32: 821-828, 2014.

22. Agelaki S, Spiliotaki M, Markomanolaki H, Kallergi G, Mavroudis D, Georgoulias V and Stournaras C: Caveolin-1 regulates EGFR signaling in MCF-7 breast cancer cells and enhances gefitinib-induced tumor cell inhibition. Cancer Biol Ther 8 : $1470-1477,2009$
23. Li ZJ, Liu M, Dawuti G, Dou Q, Ma Y, Liu HG and Aibai S: Antifungal activity of gallic acid in vitro and in vivo. Phytother Res 31: 1039-1045, 2017.

24. Lou L, Yu Z, Wang Y, Wang S and Zhao Y: c-Src inhibitor selectively inhibits triple-negative breast cancer overexpressed vimentin in vitro and in vivo. Cancer 109: 1648-1659, 2018

25. Bruzzese F, Di Gennaro E, Avallone A, Pepe S, Arra C, Caraglia M, Tagliaferri $\mathrm{P}$ and Budillon A: Synergistic antitumor activity of epidermal growth factor receptor tyrosine kinase inhibitor gefitinib and IFN-alpha in head and neck cancer cells in vitro and in vivo. Clin Cancer Res 12: 617-625, 2006.

26. Zhang T, Ma L, Wu P, Li W, Li T, Gu R, Dan X, Li Z, Fan X and Xiao Z: Gallic acid has anticancer activity and enhances the anticancer effects of cisplatin in non-small cell lung cancer A549 cells via the JAK/STAT3 signaling pathway. Oncol Rep 41: 1779-1788, 2019.

27. Phan AN, Hua TN, Kim MK, Vo VT, Choi JW, Kim HW, Rho JK, Kim KW and Jeong Y: Gallic acid inhibition of Src-Stat3 signaling overcomes acquired resistance to EGF receptor tyrosine kinase inhibitors in advanced non-small cell lung cancer. Oncotarget 7: 54702-54713, 2016.

28. Liao CC, Chen SC, Huang HP and Wang CJ: Gallic acid inhibits bladder cancer cell proliferation and migration via regulating fatty acid synthase (FAS). J Food Drug Anal 26: 620-627, 2018.

29. Yang H, Wu J, Zhang J, Yang Z, Jin W, Li Y, Jin L, Yin L, Liu H and Wang Z: Integrated bioinformatics analysis of key genes involved in progress of colon cancer. Mol Genet Genomic Med 7: e00588, 2019.

30. Chen J, Elfiky A, Han M, Chen C and Saif MW: The role of Src in colon cancer and its therapeutic implications. Clin Colorectal Cancer 13: 5-13, 2014

31. Hurwitz H, Fehrenbacher L, Novotny W, Cartwright T, Hainsworth J, Heim W, Berlin J, Baron A, Griffing S, Holmgren E, et al: Bevacizumab plus irinotecan, fluorouracil, and leucovorin for metastatic colorectal cancer. N Engl J Med 350: 2335-2342, 2004

32. Xie G, Peng Z and Raufman JP: Src-mediated aryl hydrocarbon and epidermal growth factor receptor cross talk stimulates colon cancer cell proliferation. Am J Physiol Gastrointest Liver Physiol 302: G1006-G1015, 2012.

33. Atmaca A, Werner D, Pauligk C, Steinmetz K, Wirtz R, Altmannsberger HM, Jäger E and Al-Batran SE: The prognostic impact of epidermal growth factor receptor in patients with metastatic gastric cancer. BMC Cancer 12: 524, 2012.

34. Nam B, Rho JK, Shin DM and Son J: Gallic acid induces apoptosis in EGFR-mutant non-small cell lung cancers by accelerating EGFR turnover. Bioorg Med Chem Lett 26: 4571-4575, 2016.

35. Liu ZM and Huang HS: As2O3-induced c-Src/EGFR/ERK signaling is via Sp1 binding sites to stimulate p21WAF1/CIP1 expression in human epidermoid carcinoma A431 cells. Cell Signal 18: 244-255, 2006.

36. Sen B, Saigal B, Parikh N, Gallick G and Johnson FM: Sustained Src inhibition results in signal transducer and activator of transcription 3 (STAT3) activation and cancer cell survival via altered Janus-activated kinase-STAT3 binding. Cancer Res 69: 1958-1965, 2009.

37. Chen YJ, Lin KN, Jhang LM, Huang CH, Lee YC and Chang LS: Gallic acid abolishes the EGFR/Src/Akt/Erk-mediated expression of matrix metalloproteinase-9 in MCF-7 breast cancer cells. Chem Biol Interact 252: 131-140, 2016.

This work is licensed under a Creative Commons Attribution-NonCommercial-NoDerivatives 4.0 International (CC BY-NC-ND 4.0) License. 